\title{
NeuroSky Mindwave BCI System: To Save Lives during Transportation
}

\author{
Pradnya Patil ${ }^{1}$, Dimple Chaudhari ${ }^{2}$ \\ ${ }^{1}$ Student at Mumbai University, Yadavrao Tasgaonkar Institute of Engineering and Technology, Chandhai, Karjat \\ ${ }^{2}$ Assistant Professor atMumbai University, Yadavrao Tasgaonkar Institute of Engineering and Technology, Chandhai, Karjat
}

\begin{abstract}
Ordinarily biosensor technologies examining the neurophysiological activities of driver in an operational environment are adapted with unadorned measurement encounters. This research has taken birth from studies that result into 1) subjective measures wherein sudden variations cannot be detected2) vehicle-based measures in which environmental and geometrical factors of road and vehicle are involved 3) behavioral measures wherein driver state cannot be associated to driving performance 4) physiological measures wherein self-controlled operation of vehicle is absent. The research has introduced the brain wave sensor which incessantly scrutinizes neuron pattern of brain and conveys the result to BCI system. Output from BCI system is linked with predefined vigilance level and in view of that alert is given. The proposed system thus contributes to reduce number of accidents.
\end{abstract}

Keywords: Drowsy, EEG, BCI, AAA.

\section{Introduction}

According to available statistical data, over 1.3 million people die each year on the road and 20-50 million people suffer non-fatal injuries due to road accidents. Based on the police reports, the US National Highway Traffic Safety Administration (NHTSA) conservatively estimated that a total of 100,000 vehicle crashes each year are the direct result of driver drowsiness. These crashes resulted in approximately 1550 deaths, 71000 injuries and $\$ 12.2$ billion in monitory losses. In the year 2009, the US National Sleep Foundation (NSF) reported that $54 \%$ of adult drivers have driven a vehicle while feeling drowsy and $28 \%$ of them actually fell asleep. The German Road Safety Council (DVR) claims that 1 in four highway traffic fatalities are a result of momentary driver drowsiness. These statistics suggest that driver drowsiness is one of the main causes of road accidents. A driver who falls asleep at the wheel loses control of the vehicle, an action which often results in a crash either another vehicle or stationary objects. In order to prevent these devastating accidents, the state of drowsiness of the driver should be monitored [1].

The term "drowsy" is synonymous with sleepy, which simply means an inclination to fall asleep. The stages of sleep can be categorized as awake, non-rapid eye movement sleep (NREM), and rapid eye movement sleep (REM).

Electroencephalogram (EEG) is the recording of the brainwaves from the scalp. Brainwaves are produced by synchronized electrical pulses from masses of neurons communicating with each other. The neuron pattern changes depending on the human thoughts and accordingly electrical signal is generated. The brain wave sensor placed on the scalp is utilized to detect these electrical signals. This device constitutes i) a single sensor ii) reference electrode to remove ambient noise and it can detect multiple mental states simultaneously. It is a lightweight device to be worn in operational settings which is capable of moderating the number of accidents. Sensors collect and analyze the signals further converting them into packets and transmitting through
Bluetooth. Packets are received by LSS (Level Splitter Section) giving drowsy driving alert. Emerging BCI (Brain Computer Interface) systems have been designed to use a minimal number of recording channels placing on the scalp regions of interest to reduce size, weight and cost of the system and sensors without any skin preparation and gel application. Hence, a wearable and wireless dry-electrode EEG system that can allow assessment of brain activities of participants actively performing ordinary tasks is highly desirable for BCI society. The final goal of this system is to provide a preliminary vigilance monitoring of the driver's fatigue level and give some feedback for real life applications.

\section{Literature Survey}

In 1929, the German psychiatrist Hans Berger had given a historical breakthrough introducing a new neurologic and psychiatric diagnostic tool termed as Electroencephalography (EEG) [2]. Hans Berger was the founder of first Human Electroencephalogapher in which he used a "small" string galvanometer with photographic capability, provided by Edelmann. With the device, Berger was able to make permanent recordings of 1-3 min. He was able to observe EEG from a 17-year-old boy who had a trepanation while undergoing surgery for a suspected tumor in which the front parietal surface of the cortex was exposed [3]. Further, a device, called a brain computer interface (BCI) was introduced. This was the only effective way to communicate with the environment since it can read brain signals and convert them into control and communication signals [4]. A $\mathrm{BCI}$ is an artificial system that bypasses the body's normal efferent pathways, which are the neuromuscular output channels [5]. Figure 1 demonstrates this functionality. 


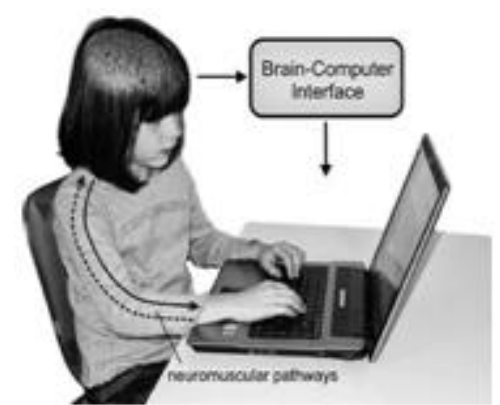

Figure 1: Brain Computer Interface

In conventional wired BCI systems, passive electrodes were widely used to measure EEG signals. To overcome the weaknesses of passive electrodes, dry electrodes were defined as those do not require the use of conductive gels or glues for installation process [6]. Recently a fourchannel based EEG device has been developed, which used electric foams to contact forehead area to obtain the brain activity. To assure the EEG signal quality, a simple validation was conducted to determine the signal distortion caused by the dry EEG sensors using a commercially available EEG system (NeuroScan Co.). The signal quality had been evaluated by examining the correlation between the EEG data simultaneously recorded by the wet and dry EEG sensors [7].

\section{Problem Definition}

As the road accidents are increasing day-by-day, it is necessary to discuss and develop a system which will avoid manual errors during driving. Though systems developed earlier those can maintain the vehicle, a hike in the number of accidents is because of drowsiness of driver. Drunk drivers also add to this number. Nowadays, a big percentile of death rate is due to accidents.

At times, accidents can occur due to blinking of eyes when driver feels sleepy. In the worst case scenario, driver may be driving with eyes open but may feel sleepy with mind. Driver's brain activities may not help driver to do proper and safe driving. This is responsible for major accidents and finally leads to death. The systems are developed which can identify the grip force on handle and can detect the sleepy state of driver. But still some systems are required which will detect the mental state of driver and will inform the driver about drowsiness.

Besides, sensors must be implemented so driver's fatigue level will get detected, but this sensor must be wearable in operational conditions. Sensors must be lightweight and comfortable enough, so that they can shoot the trouble instead of adding to it.

Moving one more step further, maintaining the vehicle in proper state during drowsy state of driver is more essential to prevent the accidents. Subsequently, the vehicle must perform self-controlled operation until awaken state of the driver.

\section{Methodology to detect drowsiness}

A new drowsiness detection system is proposed, which uses NeuroSky Mind-wave Mobile Sensor as a tool to detect the drowsiness. The brain wave sensor implemented in this system is detecting drowsiness using physiological signals, that is, EEG. Mind-wave mobile is a wireless device using a single electrode placed at forehead to record the signals and obtaining the signals using wireless Bluetooth technology. The received signals, are then converted it into small packets and forwards to LSS. LSS analyses the level and gives drowsy alert and keeps the vehicle in self-controlled function till awaken state. The proposed system constitutes three subunits which are represented as follows:

1] BCI System

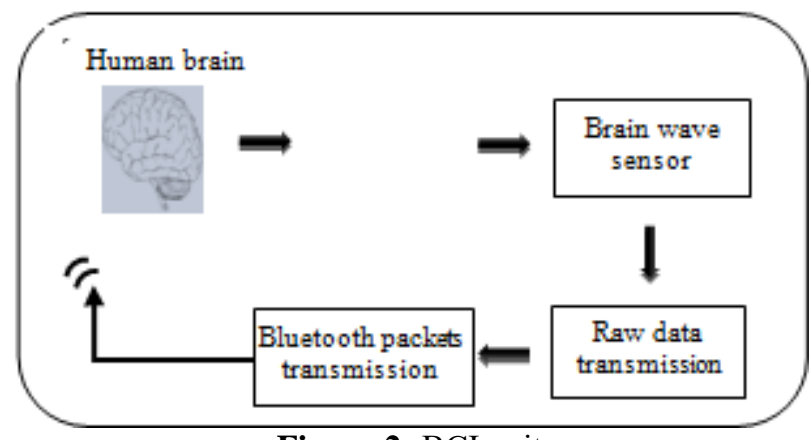

Figure 2: BCI unit

2] Data processing unit

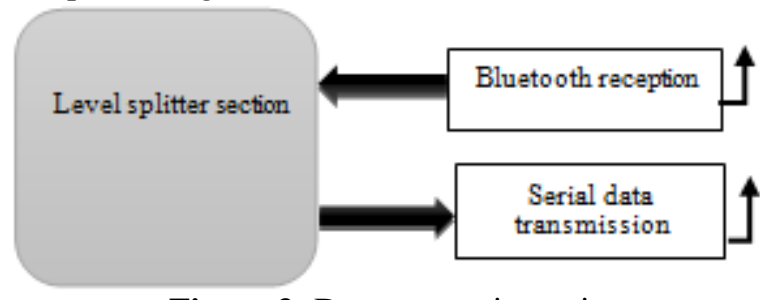

Figure 3: Data processing unit

3] Vehicle section

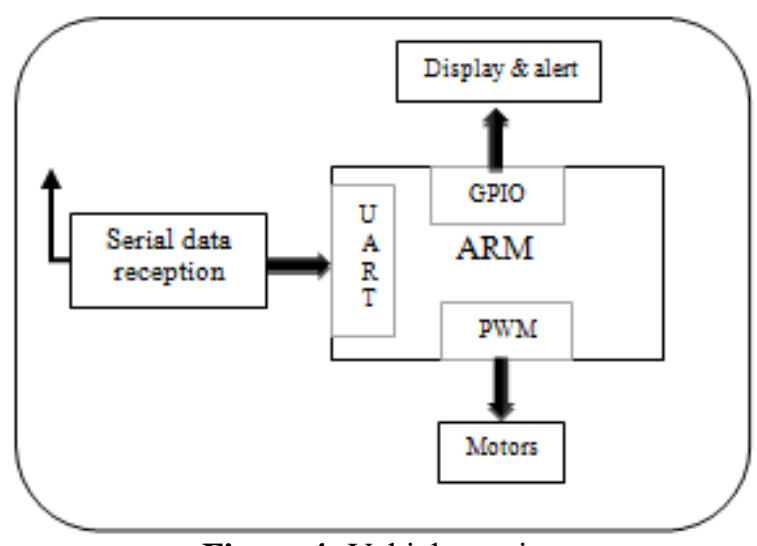

Figure 4: Vehicle section

The brain wave sensor NeuroSky Mindwave Mobile is a mobile and wireless EEG device comprising of a headset, an ear-clip and a sensor arm as shown in Figure 5. Figure 6(A). shows that a single electrode placed at FP1 position used to collect the raw brain signals. Headset's reference electrode and ground electrodes are on the ear clip as shown in Figure 


\section{International Journal of Science and Research (IJSR) \\ ISSN (Online): 2319-7064 \\ Index Copernicus Value (2013): 6.14 | Impact Factor (2014): 5.611}

6(B). Mindwave mobile offers following features exceeding the conventional wet electrodes.

a) It requires single sensor on FP1.

b) It exceeds the conventional wet electrodes with the conduction gel for long term EEG measurement.

c) It can detect multiple mental states simultaneously.

d) It has reference electrode on ear clip which will remove ambient noises.

e) It involves noise cancellation and signal amplification.

f) It provides EMG feature for eye blink detection.

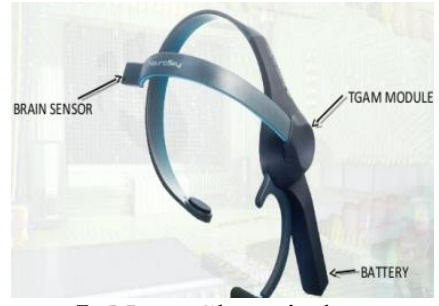

Figure 5: NeuroSky mind-wave

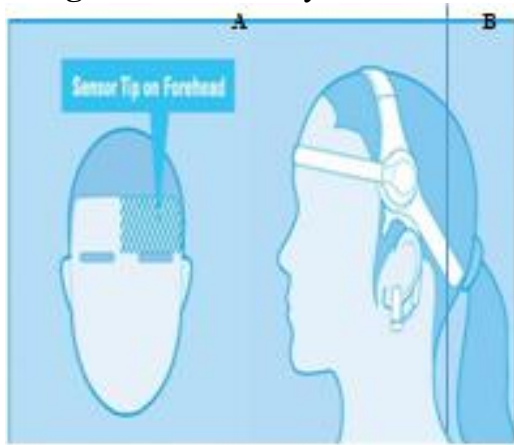

Figure 6(A): Sensor arm resting on forehead (B) Ear clip position

The mind-wave mobile weighs upto 90 grams.

- Sensor arm up is height: $225 \mathrm{~mm} \mathrm{x}$ width: $15 \mathrm{~mm} \mathrm{x}$ depth: 92mm

- Sensor Arm down: height: $225 \mathrm{~mm} x$ width: $155 \mathrm{~mm} x$ depth: $165 \mathrm{~mm}$

It requires a single AAA battery that provides 8 hours of runtime.

\section{Results}

As soon as the signal detected from brain by mind-wave mobile matches with drowsy level defined alert is given to the driver and the message "Drowsiness detected" is displayed as shown in snapshot 1 . The eSense algorithm helps to detect attention and eye blink levels which are plotted as shown in the Graph 1. Along with detection of drowsiness, self-controlled operation of vehicle is induced so that vehicle could stop by itself and accidents are avoided.

\section{Conclusion}

The eSense algorithm helps to detect attention and eye blink levels which are plotted as shown in Figure 4. Along with detection of drowsiness, self-controlled operation of vehicle is induced so that vehicle could stop by itself and accidents are avoided.

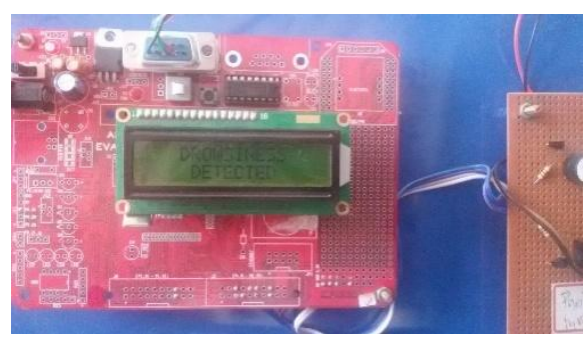

Snapshot 1

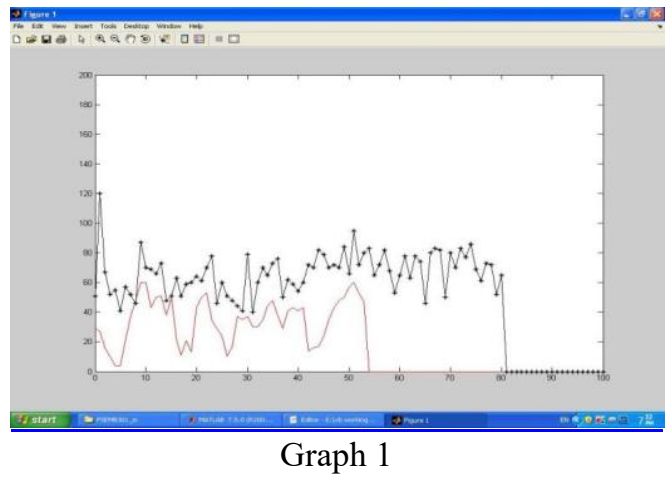

\section{Future Scope}

This research introduced a system which can give alert to driver about his own drowsiness and will stop the vehicle till awaken state of the driver. In future, the self-controlled operation of vehicle can be improved to provide more safety during driving.

\section{References}

[1] Arun Sahayadhas, Kenneth Sundaraj, and Murugappan, "Detecting Driver Drowsiness Based on Sensors: A Review".

[2] Odjel za neurokirurgiju, Klinicka bolnica Split, Split, Hrvatska, "Hans Berger (1873-1941) - The History of EEG"

[3] Thomas F. Collura "History and Evolution of Electroencephalographic Instruments and Techniques", Journal of Clinical Neurophysiology, 1993.

[4] Bernhard Graimann, Brendan Allison, and Gert Pfurtscheller, "Brain Computer Interfaces - A Gentle Introduction”, Springer-Verlag Berlin Heidelberg 2010.

[5] J.R. Wolpaw, N. Birbaumer, D.J. McFarland, G. Pfurtscheller, and T.M. Vaughan, Braincomputer interfaces for communication and control. Clin Neurophysiol, 113, Jun., 767-791, (2002).

[6] Ruijia F., Guangyuan Z., Bo C., "An on-board System For Detecting Driver Drowsiness Based on Multi-sensor Data Fusion Using Dempster-Shafer Theory", Proceedings of the International Conference on Networking, Sensing and Control; Okayama, Japan, March 2009.

[7] Chin-Teng Lin, Chun-Hsiang Chuang, Chih-Sheng Huang, Yen-Hsuan Chen, Li-Wei Ko, "Real-time Assessment of Vigilance Levelusing an Innovative Mindo4 Wireless EEG System", In Proceedings of ISCAS, 2013. 